\title{
Serial Learning: Identification of Subjective Subsequences ${ }^{1}$
}

\author{
Edwin Martin² and Davi L. Noreen \\ University of Michigan
}

\begin{abstract}
Learning of fixed arbitrary sequences proceeds by idiosyncratic subsequencing and assembly of the resulting subsequences. An error item that just precedes a subjective subsequence is a closely constrained member of that subsequence. Identification and characterization of subjective subsequences were accomplished by analyses of forward serial learning, free recall, and backward serial learning. We conclude that ordinary serial learning cannot be represented by traditional continuous-process theories, but instead must be treated by an organizational model.
\end{abstract}

When it comes to learning a sequence of events, it is clear that subjects make use of organizational principles provided by the experimenter. Thus, for example, McLean and Gregg (1967) divided a randomly ordered list of 24 letters into groups and observed differential within- and between-group interresponse times both on the criterion trial of serial recall learning and in attempts to recall the list backwardly. Similarly, Wilkes and Kennedy (1970) induced certain groupings on lists of nine letters and observed lengthened reading times at group boundaries, as well as other boundary related phenomena. Also, Lesgold and Bower (1970) have shown that when a serial list of letters is grouped into letter triplets, learning errors predominate at the position of letters that initiate a triplet.

The foregoing results exemplify the fact that subjects respond to experimenter-determined subdivision of otherwise arbitrary sequences in a serial learning task. A uscful representation of such results is that events within a group are serially organized together under a representative implicit code, that these codes are themselves grouped together in some fashion, and so on to yield a hierarchical memory structure for the sequence as a whole (Johnson, 1970; Lesgold \& Bower, 1970).

${ }^{1}$ This research was supported by the Air Force Office of Scientific Research under Contract No. F44620-72-C-0038 with the Human Performance Center, Department of Psychology, University of Michigan. The results reported herein are partially presented as Expt I in "Subjective Processes in Serial Learning," a 1973 University of Michigan honors thesis by the second author, who is now at Rockefeller University.

${ }^{2}$ Requests for reprints should be sent to Edwin Martin, Human Performance Center, 330 Packard Road, Ann Arbor, Michigan 48104.

Copyright (C) 1974 by Academic Press, Inc.

All rights of reproduction in any form reserved. 
A priori organization of a sequence is not limited to experimenterimposed grouping of otherwise mutually unrelated events. Simpson (1965), for example, controlled the serial arrangement of the same 30 words so that from condition to condition the arrangements variously approximated English word order. With random orders of words no group boundaries were evident in the bowed serial position curve. With orders that closely approximated English, however, definite boundaries were observed, corresponding to English phrase structure. The role of natural language in imposing organization on sequence learning has been welldocumented by Johnson (1968). Again, the sensible representation seems to be hierarchical in nature, in this case in terms of implicitly understood grammatical structure.

The final source of sequence organization we will mention is the organization that derives from generative production rules. The phenomenon of interest here is that when subsequences of a sequence are producible by applying different rules, errors accumulate at the position that initiates a given rule, then decline throughout the subsequence to which the rule applies. Restle (1970; Restle \& Brown, 1970) has analyzed such situations thoroughly, particularly for light-location sequences and sequences of tones in melodies. The theoretical representation he proposes entails subsequences that are dominated by generative rules (ascending, descending, trill), where these rules in turn are hierarchically dominated by further rules (transposition, repetition, mirror image). A similar orientation has been taken by Egan (1973), who has demonstrated the power of assuming a hierarchical subgoal structure for learning to solve sequential problems. In this case, errors are most frequent just after a subgoal has been attained and decline as the subject gets closer to the next subgoal.

The general picture, then, is that when subjects are faced with learning a sequence that is serially inhomogeneous, they divide the sequence into subsequences. Ilow they do the division depends on the organizational principles that characterize the sequence. On the other hand, in contrast to the progress being made toward understanding how sequences with a priori structure are learned, we have the traditional topic of serial learning wherein the sequence of events is by design as internally homogeneous as the experimenter can make it. Since the time of Ebjinghaus' germinal monograph in 1885 , most researchers have sought to remove from their sequences all bases for subdivision. An interesting concomitant fact is that the attendent theorizing about how subjects learn such sequences has generally presumed that intrasequence homogeneity was attained, both objectively and subjectively. That is, sequence construction by experimenters and theoretical hypotheses by theorists have depended on the 
notion that ordinary serial learning does not involve intralist structural heterogeneity (for reviews, see Ebenholtz, 1972; Osgood, 1953, pp. 502520; Young, 1968). For example, Ebenholtz (1972) considers briefly Mandler's (1967) general system for memory organization. Mandler's notion is that $5 \pm 2$ events is the basic limit of the organizing system. When the number of events exceeds this limit, additional groups with the same $5 \pm 2$ limits are formed. Then these groups are themselves grouped into groups. And so on until the complete set of basic events is hierarchically organized. Ebenholtz, however, intent on a unitary dimensional account of serial learning, dismisses Mandler's view by saying simply that "this approach cannot be effective in those cases where retrieval must follow a given order..." (p. 300).

From a seemingly different quarter we have the well-known facts of subject-induced organization of free-recall lists that from the experimenter's point of view have no a priori basis for subdivision (Tulving, 1962; Mandler, 1967). When given successive study-test trials on a list of unrelated events that are randomly permuted in the study phase of each trial, subjects evolve idiosyncratic groupings of those items, groupings that persist in the recall test phase even though the study phase entails rearrangement. Thus we know two things: (a) Subjective organization is a fact in tasks where the input events are scrambled from trial to trial. (b) Given any of a variety of apparent a priori bases of organization of a to-be-learned sequence, the learning progress of the subjects is heterogeneous through the sequence in ways identifiable with the organizational structure of that sequence.

From these considerations it follows that if subjects, faced with learning a sequence that from the experimenter's point of view is structurally homogeneous, in fact impose subjective organization on such sequences, then the traditional serial learning paradigm, together with all its theoretical problems, can be reassigned to the domain of organizational theories of memory. Accordingly, the purpose of the research to be reported is to identify and characterize subsequences in serial learning and thereby to deny the presumption (e.g., Ebenholtz, 1972) that ordinary serial learning can be satisfactorily understood in terms of a continuous, homogeneous theoretical representation.

\section{METHOD}

Forty-eight University of Michigan undergraduates learned a fixed serial list of 32 common four-letter words by the self-paced anticipation method. ${ }^{3}$ The words were printed on $4 \times 6$ cards. There was an initial

${ }^{3}$ The list of 32 words, in the order they were learned by all 48 subjects, is shown on the left in Figs. 1 and 2. 
inspection trial during which the experimenter showed the subject each word in succession. On anticipation trials the experimenter did not show the subject the next word until the subject either correctly anticipated it, made an error, or signaled that he was stymied. Guessing was encouraged. The trials were discrete events-the subjects could see how many cards were left in the deck as he approached the end of the list, and he waited while the experimenter tallied and informed him of the number of correct responses on that trial. This procedure continued until the subject correctly anticipated 21 out of the possible 32 words.

On completion of the serial learning task to the 21-out-of-32 criterion, the subject was given a blank sheet of paper and asked to write down as many of the words from the list as he could remember, in whatever order he happened to think of them. Following this free recall, he was asked to serially anticipate the list in backward order. There was no inspection trial-the subject started out by trying to give the last word in the list. The cards were then presented as in forward serial learning, but in reverse order. This continued until a 21 -out-of-32 criterion was met.

The only features of our procedure of any remark are that we used the same list for everyone and that we allowed the subject to proceed at his own pace. Since we have no interest in a smooth group-average serial position curve, we saw no reason to scramble the list from subject to subject; and since we did not want to obscure subjective subsequencing, we decided against pacing the subjects, which results in rushing them early in learning and disrupting them late in learning.

\section{RESULTS}

\section{Seriograms}

The basic representation of the data is in the form of what we call a seriogram. An example of one subject's seriogram is shown in Fig. 1. The numbers on the left are the 32 serial positions. Next to each number is the word that occupied that position. The latticelike display of connected dots just to the right of the words, the display labeled SL(F), represents this subject's performance in forward serial learning. A dot is a correct anticipation of a given word (row) on a given trial (column). Thus the word at Position 6 was correctly anticipated on Trials 2 through 7, while its neighbors, the words at Positions 5 and 7 , were never correctly anticipated. The word at Position 16 was correctly anticipated on Trials 3 and 4 and on Trials 6 and 7 . And so on.

There are several aspects of this seriogram worth noting. The singleword strings at Positions 6 and 12 are notable in that this subject did not enlarge on either of these groups during his seven trials to criterion. The 


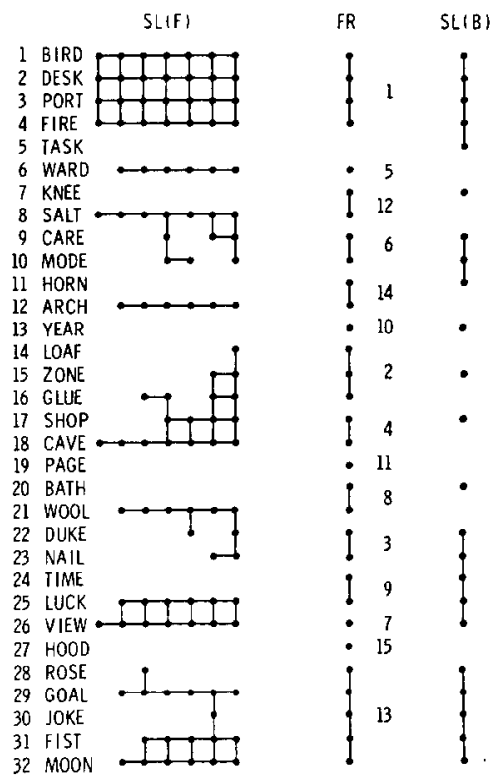

Frg. 1. Illustrative seriogram. $\mathrm{SL}(\mathrm{F})$ is forward serial learning; FR is free recall; $\mathrm{SL}(\mathrm{B})$ is backward serial learning.

triangular group that runs from Position 14 to Position 18 is a group that is being enlarged by adding on preceding words. The two groups beginning at Positions 8 and 21 are developing by addition of succeeding words.

The single column of dots under the designator FR is this subject's free recall record. A dot at a given position means that that word was recalled. Dots connected by a line represent words recalled together in a group. (We will define these groups later. For now it suffices that they represent output clusters.) The numbers to the right of these free-recall results indicate output order, with isolated dots and groups of connected dots (clusters) taken with parity.

We will make several analyses of these free recall results later, but this is a convenient place to point out a particular phenomenon. Consider the words at Positions 21, 22, and 23 in the forward serial learning lattice in Fig. 1. They make up a Size 3 group. The word at Position 21 we call an $\mathrm{F}$ word because it is the first word in the group. The word at Position 20, the word that just precedes the group and was never anticipated correctly, we call an F-1 word. When we look at this subject's free-recall record we see that the $F-1$ word was recalled, and that the $F-1$ and $F$ words were recalled together (output unit 8). If 
we now look at the column labeled SL(B), the record of this subject's first (and only) backward anticipation trial, we see that the $\mathrm{F}$ word, the one at Position 21, successfully elicited what in forward serial anticipation was its $F-1$ word. A similar relational pattern between $F-1$ and $F$ words occurs at Position 7-8, 11-12, and 24-25.

In Fig. 2 is the seriogram of another subject. He clearly learned the list in distinct subsequences of equal size. In free recall this subject produced all but one of the 32 words, neatly packaged in groups of four. But note that the output order of these groups is well-scrambled: The first group recalled was for Positions 1-4; the second was for Positions 17-20; and so on. In a postexperimental interview, this subject produced eight unrelated sentences, each containing four successive words from the list.

Note also in Fig. 2 the relation between $F-1$ and $F$ words discussed earlier for Fig. 1. At positions 17-18, 21-22, and 29-30 the group-preceding $F-1$ and group-leading-edge $F$ words are produced adjacently in free recall, and in backward anticipation the $F-1$ words are elicited by their affiliated $\mathrm{F}$ words.

Seriograms can be stored on tape or on punched cards and printed out automatically. They can be read by computer for whatever relations are

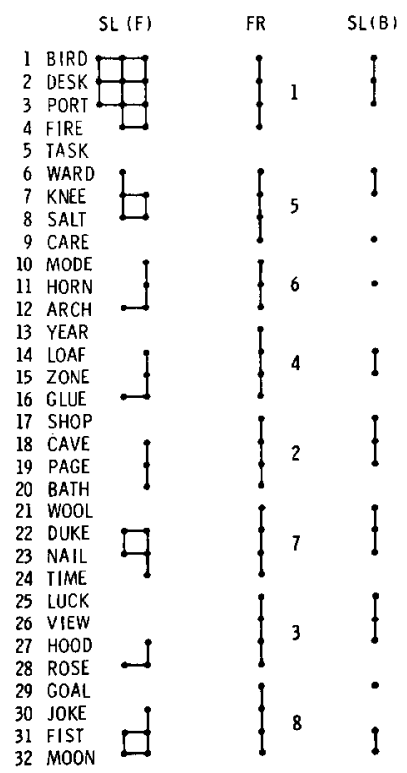

FIG. 2. Illustrative seriogram. SL(F) is forward serial learning; FR is free recall; $\mathrm{SL}(\mathrm{B})$ is backward serial learning. 
of interest. But they should be studied individually. One thing apparent from the study of individual seriograms is that there is clear subjective subsequencing. Another thing is that the subsequences are largely idiosyncratic. Certainly there is commonality over subjects with respect to either end of the list, and there is a degree of agreement that certain adjacent words in our list go together naturally, like GLUE and SHOP in Positions 16 and 17 (the strongest instance), although the two subjects represented in Figs. 1 and 2 did not participate in this agreement nor did they agree with each other.

\section{Anticipation Errors}

A common event in learning a fixed sequence is what is called an anticipation error. Such an error occurs when a subject emits a word that is wrong for the current position in the sequence but is correct for a later position. Given that we can identify with reasonable accuracy an individual subject's subjective subsequences, we can inquire into the typical source of such errors. For example, we may ask whether anticipation errors come from later positions where responding is correct or incorrect. If from later correct positions, are these positions subsequence leading-edge positions or internal positions? In any event, the general subsuming question is whether or not these presumed subjective subsequences are nomothetically related to a well-known serial learning phenomenon, namely, anticipation errors.

For this analysis we will define a subjective subsequence as a run of correct anticipations on the 21-out-of-32 criterion trial, and categorize anticipation errors on that trial according to the apparent subsequence structure at later positions.

Altogether, over all 48 subjects, wc observed 136 anticipation errors on the criterion trial such that each was identifiable with a later subjective subsequence of Size 2 or larger. By "identifiable" we mean that the anticipation error was either an $F-2, F-1, F, F+1$, or $L$ item in a later subsequence. An $\mathrm{F}$ item is the leading-edge word of the subsequence. An F - 1 item is the just-preceding word, a word not given as a correct response on the criterion trial. An F-2 item is the word just before the $\mathrm{F}-1$ item, and may or may not have been correct on the criterion trial. An F +1 item is the correctly anticipated word in the position just after the leading-edge $\mathrm{F}$ item. An $\mathrm{L}$ item is the last, or trailing-edge, word of the subsequence. It is identical to the $F+1$ item in subsequences of Size 2. Thus each of the 136 anticipation errors was classifiable as to source, where "source" means F-2, F-1, F, F + 1, and L items of subsequences of Size 2 or larger later in the criterion trial.

For each subsequence size separately we counted the number of times 


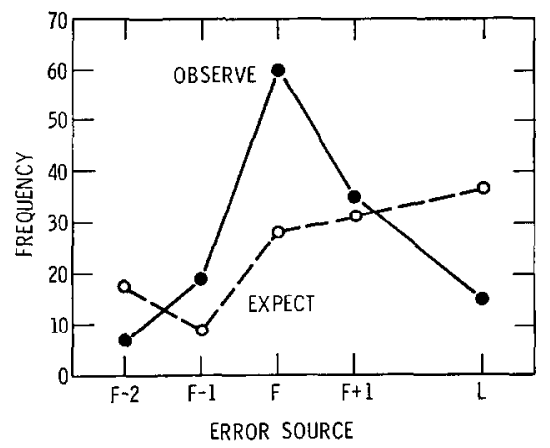

FIG. 3. Observed and expected frequency of $F-2, F-1, F, F+1$, and $L$ words as anticipation errors.

$F-2, F-1, F, F+1, \ldots$, and $L$ items were correct anticipations on trials prior to the criterion trial. This yields an expectation distribution of sorts, a distribution that can be taken to index the availability of items for responding. The open-circles dashed-line curve in Fig. 3 shows how the 136 anticipation errors should be distributed over $F-2, F-1, F$, $F+1$, and $L$ sources according to this availability expectation. The filledcircles solid-line curve shows how anticipation errors were in fact distributed. ${ }^{4}$ The trends shown in Fig. 3 are typical of all subsequence sizes.

As is evident, anticipation errors are primarily leading-edge $\mathrm{F}$ items from subjective subsequences later in the list. Items following $\mathrm{F}$ items in a subsequence are progressively less likely as anticipation errors, a trend exactly in reverse of their availability as inferred from prior correct responding. Finally, since later we will make much of the F-1 items, we note that they are a minimal source of anticipation errors.

\section{A Definition of Subjective Subsequences}

The definition of a subjective subsequence used in the preceding section (a run of correct response on the criterion trial) is but one of a set of possible definitions. It was convenient for the intended purpose. Most alternative definitions yield the same subsequences, differing only to a minor degree in whether or not a given item will be included at one or the other end of a subsequence. Since in the intertask analyses to follow we attempt to characterize the serial learning subsequences in terms of

${ }^{4}$ Since for groups of Size 2 the $F+1$ word is the $\mathrm{L}$ word, the expected and observed frequencies for Size-2 groups were repeated exactly as contributions to the $F+1$ and $\mathbf{L}$ points in Fig. 3, as opposed to making some other adjustment for opportunity. 
performance in the free recall and backward learning tasks, we settled on the following more stringent definition, which includes an item in a subsequence if it was produced with that subsequence either on the criterion trial or on two or more previous trials. We define $\{F, F+1, \ldots$, L ) as a subjective subsequence if the affiliated $F-1$ item was never a correct anticipation and $\mathrm{F}, \mathrm{F}+1, \ldots, \mathrm{L}$ were adjacent correct anticipations either on the 21-out-of-32 criterion trial or on at least two preceding trials. This definition produced 213 subsequences over the 48 subjects. Of these, 67 were Size 1 (consisting of an F item only, bounded on either side by errors) and 146 were Size 2 or larger.

\section{Free Recall}

In Table 1 is shown the proportion of $\mathrm{F}-2, \mathrm{~F}-1, \mathrm{~F}$, and $\mathrm{F}+1$ items that were free recalled. The F-2 items are those that precede the never-correctly-anticipated $\mathrm{F}-\mathrm{I}$ items and, accordingly, are presumably not identified with the subsequence $\{F, F+1, \ldots, L\}$. For the 67 Size-1 subsequences, recallability of affiliated $F-1$ items, leadingedge $F$ items, and would-be " $F+1$ " items is inferior to the recallability of the corresponding items from the 146 subsequences of Size 2 and larger. The recallability of $F-1$ items can be viewed in either of two ways: They are less available than their adjacent $F$ items, or they are seemingly readily available in light of their (definitional) absence in forward serial learning.

\section{Organization in Free Recall}

The "reality" of the subsequences identified in the serial learning task can be indexed by what clusters with what in free recall. Consider first the combination of $F-2, F-1$, and $F$ itcms. Of the 213 identified subsequences there were 135 where in free recall all three of these items were recalled. Taking only the 72 cases where the medial $F-1$ item was recalled adjacently to either its $\mathrm{F}-2$ item or its $\mathrm{F}$ item but not both, it clustered with the $\mathrm{F}$ item 63 times and with the $\mathrm{F}-2$ item only 9 times.

TABLE 1

Proportion Free Recall of Items Affiliated with Subsequences Identified in Serial Learning

\begin{tabular}{cccccc}
\hline \multirow{2}{*}{$\begin{array}{c}\text { Subsequence } \\
\text { size }\end{array}$} & $N$ & $\mathrm{~F}-2$ & $\mathrm{~F}-1$ & $\mathrm{~F}$ & $\mathrm{~F}+1$ \\
\hline & $N$ & .84 & .67 & .80 & .79 \\
$>1$ & 67 & .84 & .81 & .92 & .94 \\
\hline
\end{tabular}


Thus given that the never-correctly-anticipated $\mathrm{F}-1$ item clustered with either $\mathrm{F}-2$ or $\mathrm{F}$ in free recall, it clustered with $\mathrm{F} 87.5 \%$ of the time. The 63/9 ratio for all 72 cases breaks down to $19 / 1$ and $44 / 8$ for Size-1 and Size-2-and-larger subsequences, respectively.

Consider now the same analysis one step to the right. Of the 213 subsequences identified in serial learning, 138 involved joint recall of $F-1$, $\mathbf{F}$, and $\mathbf{F}+1$ items. For the 31 cases involving Size- 1 subsequences $F+1$ is the item that would belong to the subsequence if the subsequence were Size 2 or larger. Of the 31 cases, there were 20 in which the $\mathrm{F}$ item clustered either with its $F-1$ item or its would-be $F+1$ item but not both. The observed ratio is $20 / 0$. Thus the isolated $F$ item clustered only with its affiliated F-1 item. Of the 107 cases where the subsequence was Size 2 or larger, there were 51 cases where the $F$ item clustered with only one or the other of the $F-1$ and $F+1$ items. Here the ratio is 29/22, indicating an indifference between $F$ affinities for $F-1$ and $\mathrm{F}+1$ items.

The foregoing analyses indicate that never-correctly-anticipated F - 1 items (errors in serial learning) are closely integrated with the subjective subsequences they precede, a fact that agrees with the high incidence of errors at serial positions that initiate an experimenter-imposed subsequence (e.g., Restle, 1970).

We will report one additional fact from the free recall protocols that helps to clarify the structural relation between $F-1$ items and the subsequences with which they are affiliated. This fact has to do with the order of recall within an observed cluster. When $F-1$ and $F$ items occur together in a recall cluster, the proportion of them where the output order is $\mathrm{F}-1 \rightarrow \mathrm{F}$ is .78. Thus the backward order $\mathrm{F} \rightarrow \mathrm{F}-1$ occurred only $22 \%$ of the time. The $78 \%$ forward order for $\mathrm{F}-1 \rightarrow \mathrm{F}$ compares favorably with the $84 \%$ forward order for $\mathrm{F} \rightarrow \mathrm{F}+1$ in recall clusters containing those items. The implication here is that not only are the never-correctlyanticipated $F-1$ items closely related to their adjacent subjective subsequences, they also appear to be sequentially constrained.

\section{Within-Versus Between-Group Output Order}

Looking again at the seriogram in Fig. 2, we see eight identifiable groups. We see also that they are recalled in a scrambled order. We can certainly index the degree of agreement between the list order of these groups and their output order in free recall. Similarly, at a lower level of representation, we can index the degree of agreement between the list order of items within a group and their output order.

In this analysis, the definition of a group in free recall must be more subjective than was our earlier definition of a subsequence in forward 
serial learning. For example, consider in Fig. 2 the second cluster in free recall, the group of four words in Positions 17 through 20. The order of recall of these four words was Position 18 first, then Position 17, then Position 19, and finally Position 20. There is no question that this is a subjective group in recall-whereas this group was second in output order, its neighbors from the original list were fourth and seventh in output order. Thus a strict adjacency criterion was not used in identifying recall groups. We feel, however, that our intuitions would prove defensible against all charges.

For each of the 48 subjects we calculated a Kendall's tau correlation coefficient for each of his recall groups of Size 2 and larger, where what was correlated is output order and list order. Thus, for example, the seriogram in Fig. 2 yielded eight such taus. Once done, we then calculated a single between-groups tau for each subject using the groups themselves as units. We then did two things. First, we determined for each subject whether the groups-ordering tau was less than, the same as, or greater than the median of his several within-group taus. The result, over 48 subjects, was that $77 \%$ were less than, $6 \%$ were the same as, and $17 \%$ were greater than. Second, we calculated a composite within-group tau for each subject, which means that for each subject we added up the numerators for his several within-group taus, then the denominators, and finally divided the denominator total into the numerator total. For each subject we then compared the groups-ordering tau with the resulting composite within-group tau. What we found was that the groups-ordering tau was smaller for $83 \%$ of the subjects, the same for $2 \%$, and greater for $15 \%$. (The first way weights all groups equally, regardless of size. The second way weights groups in direct proportion to their size.)

The result of this analysis is clear: Within-group recall order was in greater agreement with the serial order of the list than was the recall order of groups as groups. Acquisition of sequence knowledge appears to proceed from that internal to subjective subsequences to that of ordering the subsequences themselves.

\section{Backward Anticipation}

We will restrict consideration of performance in the backward serial learning task to what happens in the region of what in forward serial learning were the leading edges of subjective subsequences. The salient fact is that on seeing $\mathrm{F}$ items in the backward task, the proportion correct elicitations of the $\mathrm{F}-\mathrm{l}$ items is .67 , a fact in clear contrast to the absence of correct forward anticipation of $F-1$ in the original forward task. With respect to subjective subsequences of Size 2 and larger, if we conditionalize the backward $\mathrm{F} \rightarrow \mathrm{F}-1$ transition probability on whether 
or not $\mathrm{F}$ was itself correctly elicited by $\mathrm{F}+1$, the respective values are .65 and .68 , based on 108 and 38 observations. Moreover, with respect to subjective subsequences of Size 1, where there is no F + 1 item, the backward $\mathrm{F} \rightarrow \mathrm{F}-1$ transition probability is .69 , based on 67 observations. Thus the relation between $F-1$ and $F$ items does not depend on the size of the subjective sequence nor does it depend on whether or not the link between $F$ and $F+1$ in Size 2 or larger groups is of sufficient strength for the backward $\mathrm{F}+1 \rightarrow \mathrm{F}$ transition.

That the foregoing conclusion is not vacuous can be demonstrated as follows. A subjective subsequence of Size 2 or larger involves, by definition, a never-correctly-anticipated $F-1$ item followed by $F$ and $F+1$ items. One of the backward anticipation phenomena reported above is that when $F+1$ correctly elicits $F$, then $F$ correctly elicits $F-1$ with probability .65. Now consider all subjective subsequences such that in addition to $\mathbf{F}-1, \mathbf{F}-2$ was also never correct in forward serial learning. Of these, there were 37 cases where in the backward task F correctly elicited $\mathrm{F}-1$. Here, however, the probability of the backward $\mathrm{F}-1 \rightarrow$ F -2 transition is only .24 ( 9 out of 37 ). Thus the backward elicitation capacity extends to $\mathrm{F}-\mathrm{I}$ items but not to $\mathrm{F}-2$ items. This result joins the clustering result in arguing for the $F-1$ item as an integral part of a subjective subsequence.

\section{DISCUSSION}

What we take our analyses to reveal may be summarized as follows: When faced with learning a structurally homogeneous sequence, subjects develop idiosyncratic subsequences that are easily identifiable and that sensibly relate to such other phenomena as anticipation errors during acquisition and clustering in later free recall. Moreover, certain errors in serial learning are clearly integrated members of the subsequences they precede. What this adds up to is repudiation of continuous-process theories of serial learning. Thus the following comments by Bower (1970, p. 41) are as cogent to the ordinary serial learning situation as to other areas of memory research:

The first general idea is that a preferred strategy of the adult human in learning a large body of material is to "divide and conquer"; that is, subdivide the material into smaller groups by some means, and then learn these parts as integrated packets of information. . . . The advantage of a simple grouping strategy is that the operation can be applied recursively, aggregating together chunks and then groups of chunks into an organized hierarchy.

This also was Mandler's (1967) point. The conclusion of the present writers is that the same principles of subjective organization apply to the 
traditional serial learning paradigm and therefore that speculation on how serial learning works should be subsumed under an organizational approach.

Regarding specific continuous-mechanism theories, Restle and Brown (1970) rejected the associative chaining theory of serial learning on the grounds that it could not handle learning of the branching sequences they used. We further reject it here on the grounds that even with nonbranching sequences it cannot explain the distinctive subsequencing observed in individual seriograms. Restle and Brown rejected the serial position (locational) hypothesis because group-average error profiles over serial positions are jagged in a way that agrees with a priori structural heterogeneity. We concur in this rejection, adding that if jagged serial position curves cannot be found by looking at group averages, they can be found by examining individual seriograms.

There are three likely objections to the bases of our conclusions. One is that in our experiment the subjects could pace themselves through the list in any way they saw fit, thus providing opportunity for subjective subsequencing that might not obtain in an experimenter-paced task. We cannot at present reject this possibility directly. We can, though, point out that in Simpson's (1965) study, where approximation to English word order was varied, anticipation learning was paced at a 2 -sec rate, yet the effects of implicit grammatical structure showed clearly. Similarly, rigid pacing does not obscure subsequence effects in learning rule-generated lists (Restle \& Brown, 1970). Thus in situations where a priori structure can he assumed, pacing does not prevent utilization of that structure. Moreover, in free-recall learning experiments where subjective organization emerges over trials, list presentation is typically paced, sometimes at a rate as fast as $1 \mathrm{sec}$ per item (Tulving, 1962). What these various results mean to us is that forced pacing in serial anticipation learning probably does not preclude subjective subsequencing.

The second likely objection is that we stopped our subjects short of perfect performance and based our identification of subjective subsequences largely on what we observed on the final trial, thus raising the possibility that some undetermined number of these subsequences are chance vents. In response we assert that not many of these identifications can be a mistake because (a) except for items at either end of a subsequence, a given subsequence is highly predictable from what is observable on the preceding trial, and (b) the subsequences so identified are strongly related to performance characteristics in free recall and in backward anticipation learning and to the source of anticipation errors during learning.

The final likely objection we shall treat is that having stopped our 
subjects at a subperfect stage of learning, we cannot know that the subjects' terminal knowledge of the sequence at complete mastery would in any way involve subjective subsequences. Against this possibility we can report that when the sequence contains arbitrary, experimenterimposed structure and learning is taken to a criterion of perfect performance, it is true that in ordinary leisurely free recall the subjects reproduce the list in essentially perfect serial order, but in free recall under speed stress the output order falls apart so that order of recall of the arbitrary subsequences follows a bowed serial position function (Martin, in press). Similarly, McLean and Gregg (1967) report that interresponse times in serial recall faithfully reflect subsequence organization during learning, even when serial recall is perfectly ordered. Lesgold and Bower (1970) have shown that after complete mastery of a subgrouped sequence, progress in a paired-associate task is heterogeneous according to whether the pairs do or do not span a group boundary. In other words, we have no reason to believe that subsequences clearly present at premastery stages of learning disappear upon mastery.

Contemplation of subjective structures in serial learning is not an activity that induces theoretical satisfaction. It is true that hierarchical representations appear useful and attractive (Johnson, 1970; Lesgold \& Bower, 1970; Restle, 1970; Egan, 1973), particularly on the proviso that the theorist has an a priori idea as to what the effective structure might be. In the case of serial learning of a homogeneous sequence, subjects not only make up their own subsequences, they also enlarge them from trial to trial, sometimes by addition of items on the front end of a subsequence, sometimes by addition on the back end. Thus subsequence size as well as boundary location must be random variables at the outset, and both must change probabilistically over learning trials.

\section{REFERENCES}

Bower, G. H. Organizational factors in memory. Cognitive Psychology, 1970, 1, 18-46. Ebbinghaus, H. Über das Gedächtnis. Leipzig: Ducker \& Humbolt, 1885. (Transl. H. A. Ruger \& C. E. Bussenius. New York: Dover, 1964.)

Ebenhor.tz, S. M. Serial learning and dimensional organization. In G. H. Bower (Ed.), The psychology of learning and motivation, Vol. 5. New York: Academic Press, 1972.

EGaN, D. E. The structure of experience acquired while learning to solve a class of problems. Unpublished doctoral thesis, University of Michigan, 1973.

Johnson, N. F. Sequential verbal behavior. In T. R. Dixon \& D. L. Horton (Eds.), Verbal behavior and general behavior theory. Englewood Cliffs: Prentice-Hall, 1968.

Johnson, N. F. The role of chunking and organization in the process of recall. In G. H. Bower (Ed.), The psychology of leaming and motivation, Vol. 4. New York: Academic Press, 1970. 
LesGold, A. M., \& Bower, G. H. Inefficiency of serial knowledge for associative responding. Journal of Verbal Learning and Verbal Behavior, 1970, 9, 456-466.

Martin, E. Serial learning: A multilevel access analysis. Memory \& Cognition, in press.

Mandler, G. Organization and memory. In K. W. Spence \& J. T. Spence (Eds.), The psychology of learning and motivation, Vol. 1. New York: Academic Press, 1967.

McLear, R. S., \& GregG, L. W. Effects of induced chunking on temporal aspects of serial recitation. Journal of Experimental Psychology, 1967, 74, 455-459,

Osgood, C. E. Method and theory in experimental psychology. New York: Oxford University Press, 1953.

Restle, F. Theory of serial pattern learning: Structural trees. Psychological Review, 1970, 77, 481-495.

Restle, F., \& Brown, E. R. Serial pattern learning. Journal of Experimental Psychology, 1970, 83, 120-125.

Simpson, W. E. Effects of approximation to sentence word-order and grammatical class upon the serial liarning of word lists. Journal of Verbal Learning and Verbal Behavior, 1965, 4, 510-514.

Tulving, E. Subjective organization in free recall of "unrelated" words. Psychological Review, 1962, 69, 344-354.

WiLkes, A. L., \& Kennedy, R. A. The relative accessibility of list items within different pause-defined groups. Journal of Verbal Learning and Verbal Behavior, 1970, 9, 197-201.

Young, R. K. Serial learning. In T. R. Dixon \& D. L. Horton (Eds.), Verbal behavior and general behavior theory. Englewood Cliffs: Prentice-Hall, 1968.

(Accepted January 11, 1974) 